\title{
MONITORAMENTO DA TEMPERATURA DA ÁGUA NAS BOMBAS CENTRÍFUGAS NOS VEÍCULOS DE COMBATE A INCÊNDIO
}

\author{
Paulo César Macedo ${ }^{1}$ \\ Licurgo Borges Winck ${ }^{2}$
}

\section{RESUMO}

O aumento da temperatura da água em corpos de bombas centrífugas é um dos fatores causais de várias anomalias. O mesmo tem ocorrido em caminhões de combate a incêndios, quando utilizados em instrução de flash over, onde o fluido circula internamente em alta pressão propiciando aumento de temperatura. Dessa forma, este estudo objetivou monitorar a temperatura do corpo de bombas em relação ao tempo de funcionamento. Para tal propósito, foi instalado um sensor de temperatura na tubulação de retorno de água no ABT-26. Os resultados deste experimento mostraram que após 18 min de funcionamento do corpo de bomba a temperatura aumentou para $41^{\circ} \mathrm{C}$, a qual é prejudicial ao equipamento. No entanto, não houve identificação de ruídos, vibrações ou quaisquer outros alertas de cavitação, que permitisse ao operador do sistema observar o fenômeno e cessar tal atividade danosa ao equipamento. Além disso, foi observado que o fluxo de água expelido do sistema não foi suficiente para retirar o calor produzido pela bomba, uma vez que o ponto real de trabalho foi de $0,49 \%$ quando o recomendado seria de $80 \%$ da capacidade da bomba. Portanto, este trabalho mostrou ser de grande importância para o corpo de bombeiros, pois monitorando a temperatura do sistema torna-se viável evitar possíveis danos ao corpo de bombas e a integridade física do combatente.

Palavras-chave: Monitoramento. Temperatura. Cavitação. Pressão de Vapor.

\footnotetext{
${ }^{1}$ Aspirante a oficial no Corpo de Bombeiros Militar do Estado de Rondônia - CBMRO. Possui Curso Técnico em Eletromecânica pelo Instituto Federal de Ciências e Tecnologias do Estado de Rondônia, 2012, Rondônia-RO e Curso Superior em Gestão Pública pela Faculdade Educacional da Lapa, 2016, Vilhena-RO.

${ }^{2}$ Tenente do Corpo de Bombeiros Militar do Estado de Goiás. Doutor em Ciências Mecânicas. E-mail: licurgo2006@gmail.com.
} 


\title{
WATER TEMPERATURE MONITORING IN CENTRIFUGAL PUMPS IN FIRE FIGHTING VEHICLES
}

\begin{abstract}
Increasing water temperature in centrifugal pump bodies is one of the causal factors of many anomalies. The same has been the case in firefighting trucks when used in flash over instruction, where the fluid circulates internally at high pressure providing temperature rise. Thus, this study aimed to monitor the pump body temperature in relation to the operating time. For this purpose, a temperature sensor was installed in the water return pipe on the ABT-26. The results of this experiment showed that after $18 \mathrm{~min}$ of pump body operation the temperature increased to $41^{\circ} \mathrm{C}$ which is detrimental to the equipment. However, no noise, vibration or other cavitation alerts were identified that would allow the system operator to observe the phenomenon and cease such harmful activity to the equipment. In addition, it was observed that the flow of water expelled from the system was not sufficient to remove the heat produced by the pump, since the actual working point was $0.49 \%$ when the recommended would be $80 \%$ of the pump capacity. Therefore, this work proved to be of great importance to the fire department, as monitoring the system temperature makes it possible to avoid possible damage to the fire department and the physical integrity of the combatant.
\end{abstract}

Keywords: Monitoring.Temperature. Cavitation. Steam pressure.

Artigo Recebido em 17/09/2019 e Aceito em 12/03/2020 


\section{INTRODUÇÃO}

O aumento da temperatura em bombas centrífugas tem sido um problema recorrente em caminhões de combate a incêndio que são submetidos a técnica Flash over. A alta temperatura, na maioria dos sistemas mecânicos, traz grandes prejuízos, uma vez que, além dos danos mais conhecidos, como exemplo, a cavitação, que de maneira resumida é a formação de bolha na região de baixa pressão e sua implosão quando passada para a região de alta, uma série de estragos podem estar associados a temperatura.

É importante enfatizar que o objetivo do trabalho não foi apenas aferir a temperatura, que é responsável pela cavitação do líquido em questão, mas também compreender os problemas advindos do aumento da temperatura, discutir o aumento da mesma proveniente da baixa vazão, entender a relação da pressão de vapor com a cavitação, coletar dados acerca da temperatura em decorrência do tempo, e um dos mais importantes, instalar um sensor para monitorar o comportamento térmico desse fluido.

Oestudo da Cavitação, foi entendido como de suma importância, pois quando se trata de bomba centrífuga é sempre um desafio lidar com as consequências do aumento da temperatura, e no caso do Corpo de Bombeiro do Estado de Goiás não foi diferente.

Novas técnicas de combate a incêndio foram adotadas e estas, principalmente quando são executadasaos treinamentos, exige-se muito do corpo de bombas das viaturas, uma vez que, a água é mantida sob alta pressão por um longo espaço de tempo sendo que o consumo da mesma é efetivamente baixo, resultando em um grande volume circulatório e aumento de temperatura.

No que se refere a metodologia aplicada, este estudo teve uma abordagem bibliográfica e experimental, com análise qualitativa e quantitativa 
dos dados adquiridos através dos testes. A parte experimental teve início na Academia de Ensino Bombeiro Militar em Goiânia-GO, com a instalação de dispositivos na viatura de combate a incêndio.Já a coleta de dados aconteceu no Centro de Operações e Tecnologia de Incêndio em Anápolis-GO.

A estruturação deste trabalho se deu por meio de tópicos onde foram abordados conceitos relacionados às bombas centrífugas, bem como seu aquecimento. Posteriormente, os dados experimentais foram analisados através de comparações com o referencial bibliográfico.

\section{BOMBAS CENTRÍFUGAS}

Nesta seção, paraser entendido o motivo pelo qual a análise da temperatura é de suma importância para o referido trabalho, primeiramente foi posto alguns conceitos relacionados às bombas dinâmicas, sobretudo, bomba centrífuga de incêndio tipo BAC, bombas de acionamento por caixa de transferência, (Figura 1), bem como seu funcionamento, e o entendimento de alguns fenômenos.

Figura 1 - Modelo de bomba BAC

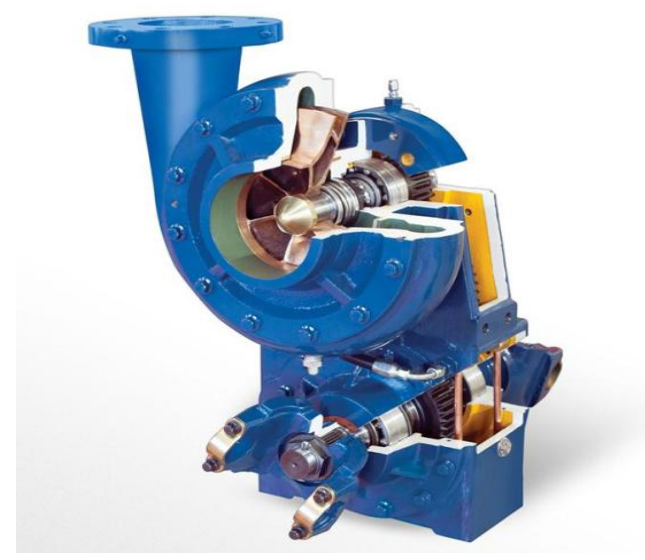

Fonte: Mitren, 2016 
As bombas dinâmicas, também conhecidas como turbo-bombas, podem ser classificadas em: bombas de fluxo misto, bombas de fluxo axial, bombas periféricas e bombas centrifugas(Gomes, 2013). Para Oliveira (2017), as centrífugas ainda podem ser classificadas em: Centrífuga radial, axial e helicoidal. Contudo, o alvo são as centrífugas radiais, uma vez que a pesquisa experimental foi voltada a ela.

A bomba centrífuga é um dispositivo que transforma energia cinética em energia hidrodinâmica, tendo como fonte de energia rotacional, por exemplo, um motor elétrico ou a combustão. As bombas centrífugas são aquelas em que a energia fornecida ao líquido é primordialmente do tipo cinética, sendo posteriormente grande parte convertida em energia de pressão(Gomes, 2013).

Vale ressaltar que as bombas centrífugas do tipo BAC modelo $\mathrm{M}-750$, equipamento estudado nessa pesquisa, são dispositivos específicos para viaturas de combate a incêndios. O que difere estas das demais é o fato de ser acoplada a uma caixa de transferência, cuja função é desviar a potência do motor para bomba (Mitren, 2016).

\subsection{Partes e Funcionamento}

Os componentes de uma bomba centrífuga, bem como sua capacidade varia de acordo com os fabricantes e modelos existentes. Segundo Gouvea (2008), esta bomba pode ser dividida em três partes principais com suas respectivas funções são: 1- rotor: propelir o fluido; 2- eixo: transmitir 0 movimento rotativo ao impelidor e 3-carcaça: dar sustentabilidade mecânica a todos os elementos da bomba, bem como conter o fluido e direcioná-lo tanto na alimentação como na descarga (Figura2). 
Figura 2 -Partes principais de uma bomba centrífuga

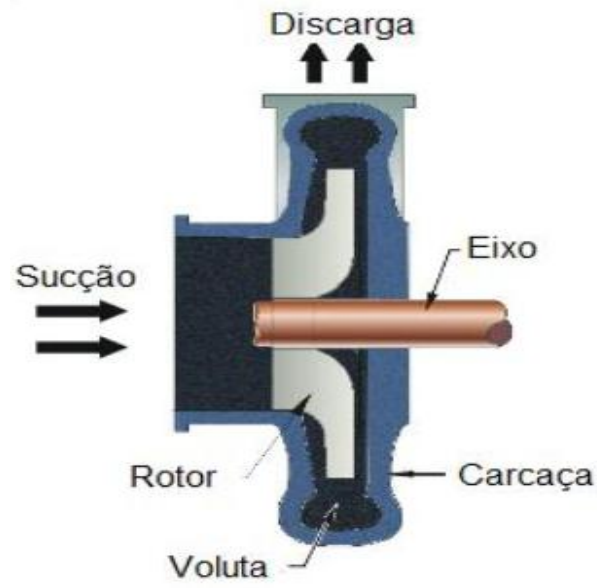

Fonte: Mitren, 2016

\subsection{Princípio de Funcionamento de BombasCentrífugas}

Segundo Souza (2014), O princípio de funcionamento das bombas centrífugas se dá pelo ganho de energia cinética do fluido no impelidor, que posteriormente é convertida em pressão.

O manual de operação da Mitren (2016) ainda explica que isso ocorre quando a água entra no rotor através do duto de admissão (Figura 3A).Processo denominado de alimentação axial, dado que, o líquido flui na direção do eixo. Dessa forma, a água que advém da sucção é limitada pelas pás do rotor sendo então defletida em $90^{\circ}$,fluindo por meio do rotor na direção perpendicular ao eixo até a câmara de descarga (Mitren, 2016). 
Figura 3 -Princípio de funcionamento

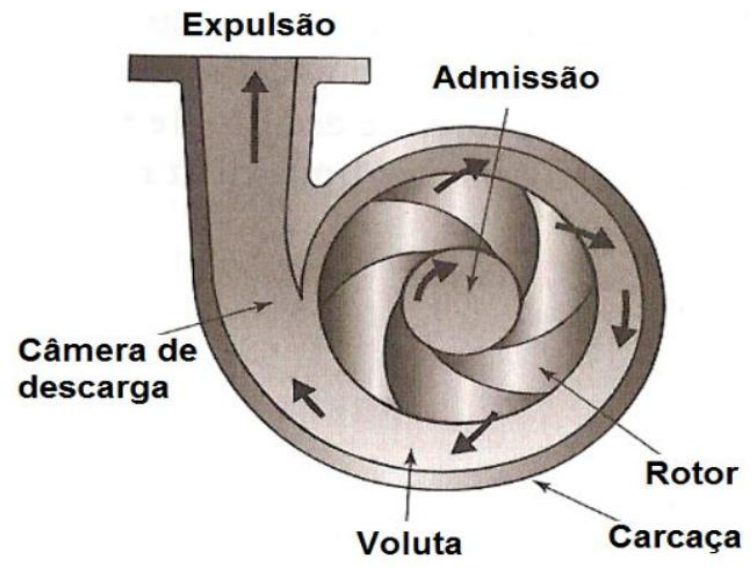

A) Princípio de funcionamento

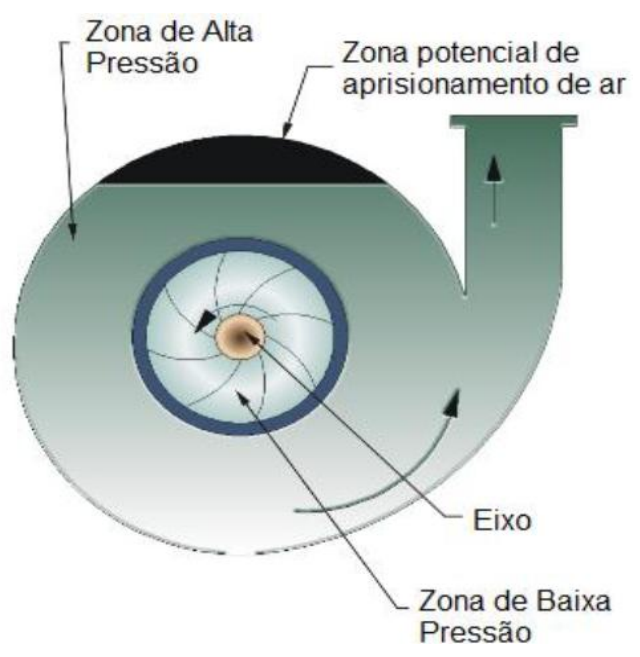

B)Zonas de Pressão

Fonte: Mitren, 2016.

A Figura 3B mostra as zonas de pressão que possuem relação direta com alguns problemas relacionados a bombas centrífugas, sobretudo a cavitação. Na entrada de água, situa-se a zona de baixa pressão, momento no qual o fluido está sendo sugado ao centro da bomba. Por outro lado, à medida que as pás do rotor comprimem o fluido contra a voluta, aumenta-se a pressão.

Existem três fatores que influenciam diretamente no desempenho de uma bomba, como velocidade angular, pressão e vazão, sendo estes interrelacionados. O manual de operações de bombas da Mitren (2016 p.9) os define como:

Velocidade angular - Se a velocidade de rotação aumentar, mantendo um fluxode fluido constante a pressão da água na saída da bomba irá aumentar. Avelocidade angular é usualmente medida em rotação por minuto (rpm). Pressão - Se a pressão mudar com a velocidade mantida constate, a vazão dofluido mudará inversamente, isto é, se a pressão aumentar, a vazão da água nadescarga diminuirá. A pressão é usualmente medida em libras por polegadaquadrada (psi), quilogramas força por centímetro quadrado $\left(\mathrm{Kgf} / \mathrm{cm}^{2}\right)$ ou quiloPascal (Kpa).Fluxo-Vazão- Se a pressão for mantida constante, a vazão aumentará com oaumento na velocidade da rotação da bomba centrífuga. A vazão é usualmentemedida em galões por minutos (gpm) ou litros por minutos (lpm). 
Dessa maneira, qualquer bomba centrífuga, cujas variáveis citadas acima não forem compatíveis com as pré-estabelecidas pelo fabricante estará passiva de graves problemas, por exemplo,danos oriundos de alta temperatura.

\subsection{Princípio de Funcionamento da Caixa de Transferência}

A caixa de transferência permite que o torque do motor seja transferido à bomba por meio de uma engrenagem seletora (Figura 4A). Neste aspecto, 0 manual da Mitrem (2016 p.4) principal fonte relacionada a caixa de transferência diz que:

A caixa de transferência das bombas BAC é do tipo "Split Shaft". Este tipo detransmissão permite transferir o torque e a rotação entre dois eixos alinhados. Atransferência da força é feita pelo deslocamento de uma engrenagem ranhurada sobreos eixos. Esta engrenagem possui duas posições de trabalho. Na primeira, ela ficaposicionada sobre os dois eixos. Assim, quando o eixo motriz gira ela transfere estemovimento ao segundo eixo, o eixo piloto. Na segunda posição a engrenagem ficaposicionada somente sobre eixo motriz, desconectando o eixo piloto, que fica livre.Nesta posição o dentado externo da engrenagem deslizante também fica engrenadocom a engrenagem intermediária da caixa de transferência, a qual transfere omovimento para o eixo da bomba.

Dito isso, se o eixo motriz estiver unido ao eixo piloto não haverá transferência de potência à bomba. Mas se a engrenagem estiver somente sobre o eixo motriz, sua parte externa estará engrenada com as outras engrenagens da caixa de transferência, o que permitirá que essas transmitem rotação e torque ao eixo da bomba centrífuga.

Figura 4 - A) Visão interna e de perspectiva lateral da caixa. B) Sistema completo: 1corpo de bomba com caixa, 2- cardam dianteiro, 3-cardam traseiro. 


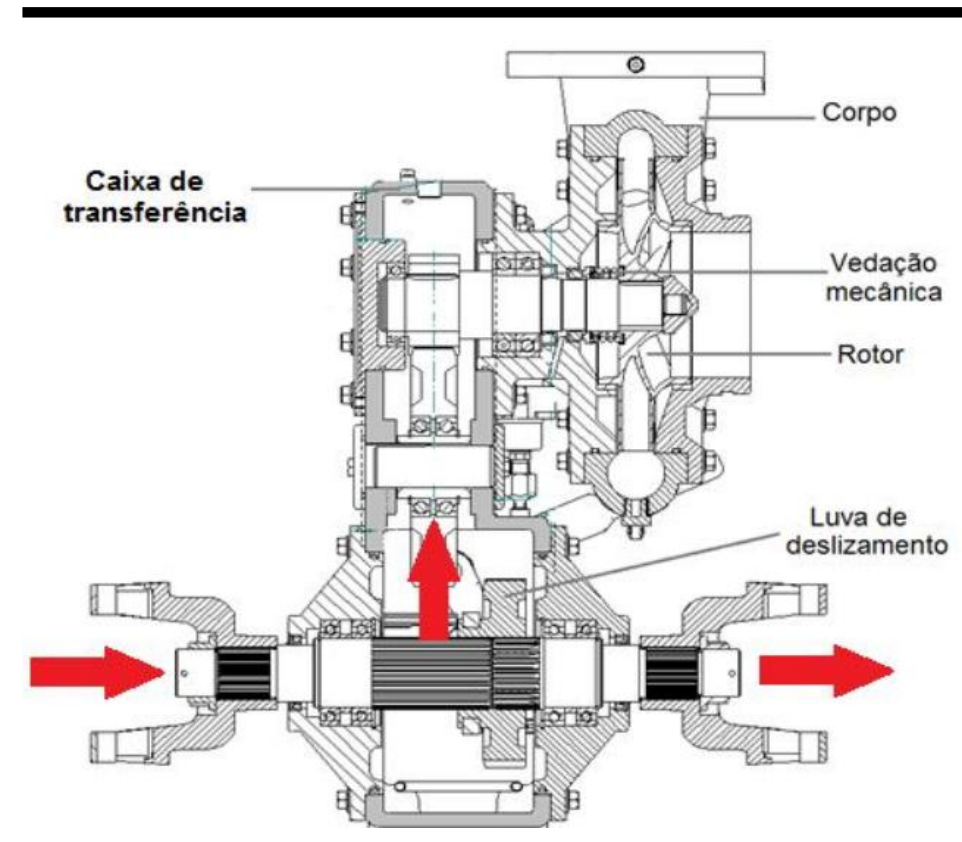

A)

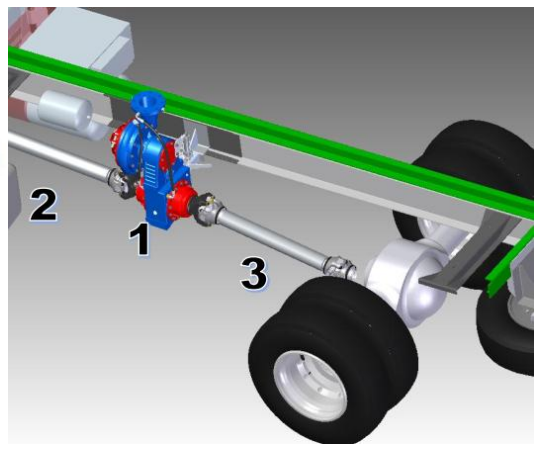

B)

Fonte: Mitren, 2016

\subsubsection{Sistema de Arrefecimento da Caixa de Transferência}

Componentes mecânicos rotativos, normalmente geram atritos e aquecem levando a necessidade de um sistema de arrefecimento. Com as caixas de transferência acopladas às bombas Mitren tipo BAC não é diferente, pois são contempladas com um trocador de calor do tipo serpentina (Figura 5A). Segundo Souza (2013)esse dispositivo pode ser definido como um equipamento em que dois fluidos em diferentes temperaturas trocam calor utilizando uma superfície metálica, no qual pode ou não ocorrer mudança de fase dos fluidos. 


\section{Revista FLAMMAE}

Revista Científica do Corpo de Bombeiros Militar de Pernambuco

Artigo Publicado no Vol.06 N.15 - Edição Jan a Jun 2020 - ISSN 2359-4829

Versão on-line disponível em: $\underline{\text { http://www.revistaflammae.com }}$

Nota-se ao analisar a Figura 5B, que é utilizado a próprio líquido bombeado para o sistema de arrefecimento. A água é coletada na região de descarga da bomba de incêndio, passa por toda a serpentina e volta para a admissão dela.

Figura 5 - A) Serpentina. B) Corpo de bomba com sistema de arrefecimento

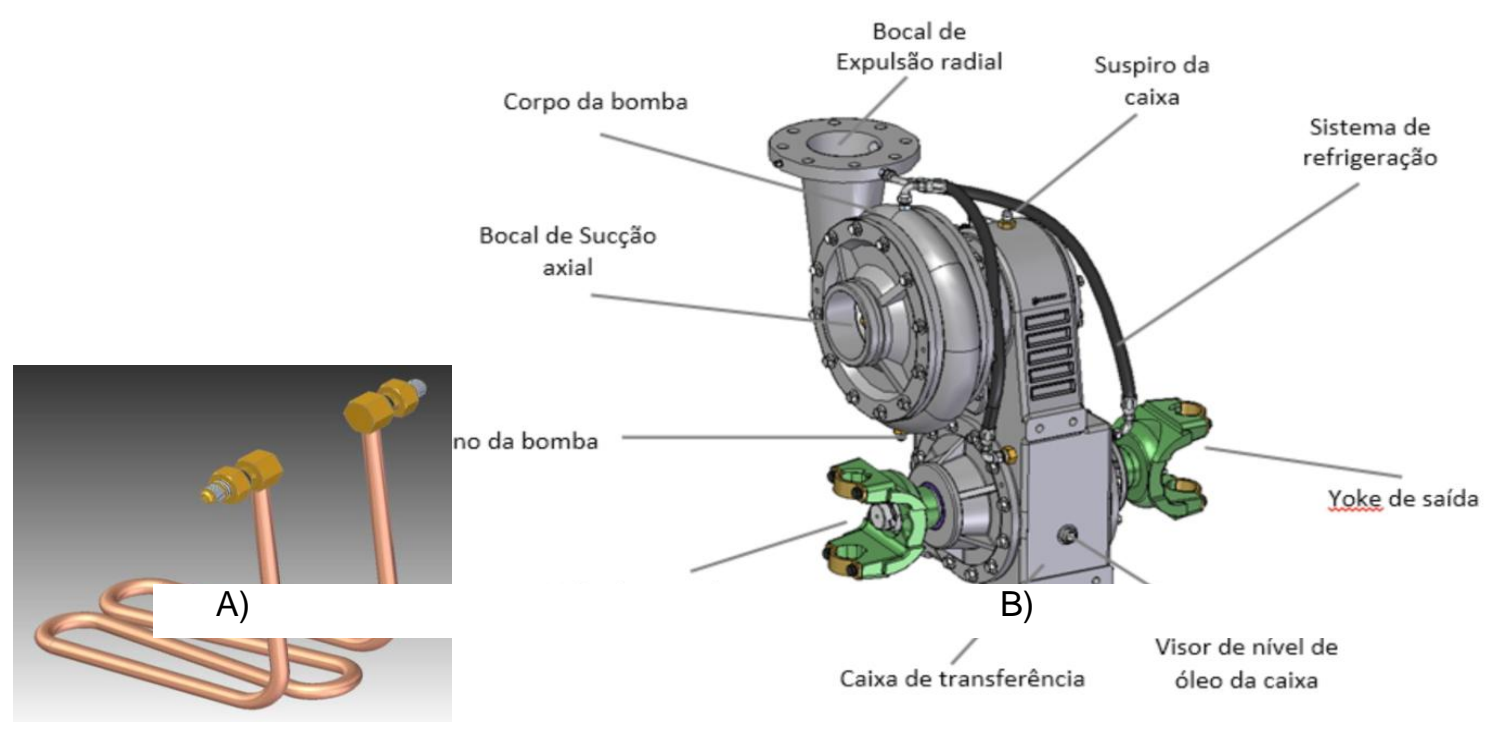

Fonte: Mitren, 2016.

\subsection{Problemas Relacionados à Temperatura}

As bombas são fabricadas de acordo com o trabalho que exercem, cada modelo disponível tem suas peculiaridades, como exemplo, ponto de melhor vazão de trabalho, garantindo a inexistência de fenômenos maléficos aos sistemas (Gouvea 2008). Para entender as consequências do aumento da temperatura em sistemas de bombas, previamente foi necessário saber as maneiras mais comuns geradora desse aquecimento, mesmo sabendo que 0 fator principal está relacionado ao funcionamento em baixa vazão. 


\subsubsection{Aumento da Temperatura Proveniente de Baixa Vazão}

Como anteriormente citado, toda bomba tem suas características nas quais torna-se mais eficiente. No caso da vazão, o estudo deGouvea (2008) atribuiu

ponto de melhor eficiência em torno de $70 \%$ a $85 \%$ da capacidade máxima para a maioria das bombas centrífugas de simples estágios. Isso porque, em vazões distantes do BEP as forças hidráulicas perdem balanceamento, resultando em baixa eficiência, maior nível de deflexão no eixo e aumento de vibrações.

Este autor também constatou que, a vazão de fluido através da bomba não é suficiente para remover o calor gerado internamente na bomba, quando submetida àvazão muito baixa.

Portanto, fica evidente que em situações na qual não forem atendidas o mínimo de vazão durante a atividade, poderá haver um superaquecimento do sistema de maneira que toda a estrutura do corpo de bombas venha ser comprometida, principalmente pelas consequências advindas da cavitação e outras anomalias semelhantes.

\subsubsection{Cavitação e Pressão de Vapor}

Para compreender melhor o fenômeno da cavitação, antes de tudo, é imprescindível assimilar o que é pressão de vapor. Segundo Gouvea (2008), pressão de vapor pode ser definida como uma medida da tendência de evaporação de um líquido. Quanto maior a sua pressão de vapor, maior é essa tendência e mais volátil fica o líquido.

Em outras palavras, Gomes (2013) diz que em um sistema hidráulico com temperatura constante, onde a pressão do líquido for maior que a pressão de vapor, haverá somente líquido. Mas se a pressão do líquido for reduzida a 
níveis iguais ou menores que a pressão de vapor, o líquido passa instantaneamente para a fase de vapor.

Nestas condições, Laranjeira (2017), afirma queo fenômeno da cavitação ocorre quando a pressão do fluido, na temperatura de bombeamento, atinge valor igual ou inferior a pressão de vapor. Sendo que, nestas circunstâncias parte do líquido irá se vaporizar e formará bolhasem uma região crítica de baixa pressão, conhecida também como olho do impelidor.

Dessa maneira, quando as bolhas passam para a zona de recalque, onde a pressão exercidasobre o líquido é maior, estas são comprimidas até que sofram implosão, e por consequência, emitem fortes ondas de choque.Assim, Gouvea (2008 p.2) diz que:

Caso a parte vaporizada encontre alguma região onde pressão absoluta seja maior que a pressão do vapor líquido na temperatura de bombeamento, haverá colapso das bolhas com retorno à fase líquida e a geração de ondas de choque, esse fenômeno é conhecido como cavitação, gerando vibrações, ruídos e danos à bomba centrífuga.

Por outro lado, (Melo 2008) discorre em seus estudos que, existem pelo menos duas formas de aumentar a pressão de vapor e, consequentemente, dar início ao fenômeno de cavitação. Sendo uma delas a diminuição da pressão do sistema, e a outra a elevação da temperatura do líquido na sucção da bomba.

Não obstante, é mister salientar sobre a recirculação de fluido em bombas centrifugas, pois, seus danos, muitas vezes são confundidos com os provenientes da cavitação, todavia tem origens diferentes, isto é, por recirculação do fluido na sucção ou na expulsão (Fraser, 1981).

\subsubsection{Temperatura Limite Tolerável}

Para concluir esse tópico, e enfatizar a influência da temperatura no desempenho do funcionamento do corpo de bomba, sobretudo no NPSH (Net Positive Suction Head Head) Coelho (2006) relata que:

Além da influência da altura de sucção e da vazão bombeada, o valor do NPSH é também influenciado pelas propriedades termodinâmicas do líquido transportado, que podem ser modificadas em um mesmo 
Assim sendo, é importante que seja relatado que existe um limite tolerável de calor, instante em que os efeitos da cavitação se tornam mais danosos em relação a erosão provocada pelo fenômeno.Segundo Feghali (2006),nos seus estudos utilizando água, a corrosão por cavitação variou com atemperatura, e ficou comprovado que atinge maior intensidade para temperatura na ordem de $45^{\circ} \mathrm{C}$. Logo, entende-se que em sistemas compostos por bombas centrífugas, é recomendável que a temperatura não ultrapasse $45^{\circ} \mathrm{C}$ para que as consequências de uma possível cavitação não sejam agravadas.

\subsection{Sensor}

De acordo com Carneiro (2009), sensor de temperatura é um instrumento capaz de sentir a temperatura e passar essa informação ao sistema descodificante, permitindo que o dado seja visualizado em um monitor. Por outro lado, existem inúmeros modelos desse dispositivo, com aplicação para diversas áreas. Um dos mais utilizados no mundo da mecânica são os termopares, cujo funcionamento é baseado no efeito de Seebeck, devido a combinação de dois metais que possuem diferença de potencialde saída previsível e abrangem alta amplitude de temperaturas (Silva, 2006).

Para os estudos realizados neste trabalho,foi utilizado termopar do tipo k, uma vez que são equipamentos de simples instalação, tem-se uma grande resistência mecânica, bem como boa precisão nas leituras e ampla faixa de abrangência, ou seja, desde temperatura negativas, na escala Celsius até altas temperaturas. 


\section{METODOLOGIA EXPERIMENTAL}

\subsection{Material e Métodos}

O experimento foi realizado na viatura de combate a incêndio (ABT-26), utilizando para a montagens de linhas mangueiras de 2", 1/2 e de 1"1/2 ambas do tipo 4, esguicho tipo pistola com bitola de entrada de 1,1/2" e um divisor de $2,1 / 2$ " para duas linhas de 1,1/2", ocorrido durante as instruções de instrutor Flash over no Centro de Operações e Tecnologia de Incêndio do Corpo de Bombeiros Militar em Anápolis - GO.

Para a verificação da temperatura, foi instalado uma conexão nipletipo "T" e um sensor termopar do tipo k na tubulaçãoque faz parte do sistema de retorno do fluido ao tanque, saída de 2"1/2 lado direito do corpo de bomba. Em referência ao sistema elétrico foi executado conforme o diagrama emitido pelo fabricante (Figura 6). Também foram instalados um monitor digital para visualização dos dados e um fusível de 10A para proteção do sistema elétrico.

Para determinar a relação da temperatura em função do tempo, o experimento foi iniciado após o acionamento do corpo de bomba e ajustes das variáveis (vazão, pressão e velocidade angular). Este estudo consistiu em monitorar grau em grau até $41{ }^{\circ} \mathrm{C}$ por aproximadamente uma hora de funcionamento da bomba dividido em três etapas. Para garantir uma reprodutibilidade dos resultados, todos os materiais e variáveis foram mantidos os mesmos em todos os experimentos. 
Figura 6 - Diagrama elétrico

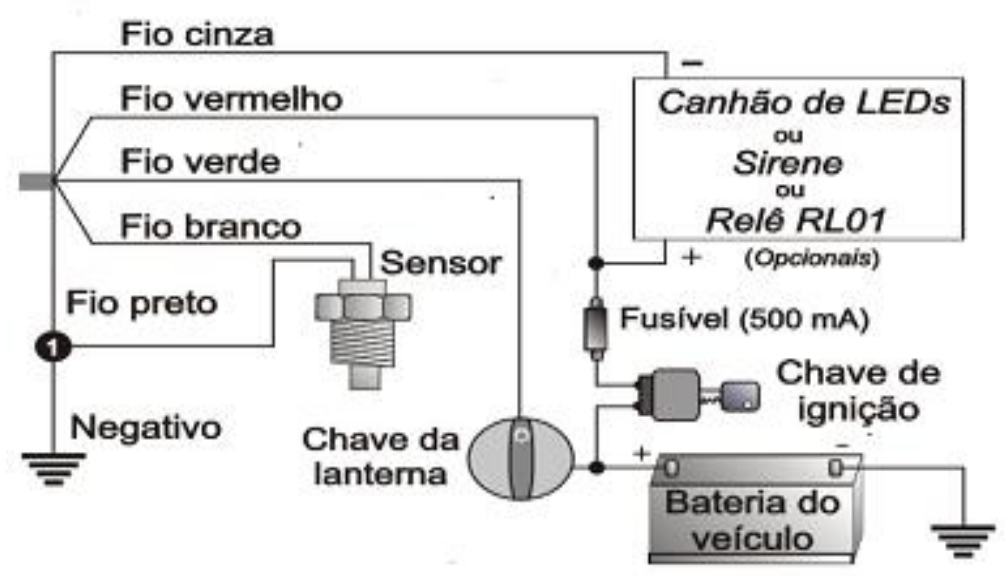

Fonte:Racetronix, 2019.

\section{RESULTADOS E DISCUSSÃO}

\subsection{Sensor de Temperatura}

Primeiramente, como um dos objetivos para saber se realmente a água aquece durante o funcionamento da bomba centrífuga do caminhão de combate a incêndio, foi necessário a instalação de um sensor de temperatura em local onde a leitura não fosse comprometida.

$\mathrm{Na}$ ocasião, foi posto um sensor termopar semelhante ao utilizado em sistema de arrefecimento veicular. Este, por sua vez, além de permitir o acoplamento de um monitor, tem faixa útil de operação entre 0 a $125^{\circ} \mathrm{C}$, capacidade de gravar a maior temperatura atingida, acionamento de alarme sonoro e luminoso quando a temperatura estabelecida como limite for atingida e funciona apenas com tensão de 12 Volts. 
No que diz respeito ao posicionamento do sensor, optou-se por colocá-lo na tubulação que antecede a boca expulsora, dado que dentre outros motivos citado no próximo tópico foi um local acessível à instalação. Por outro lado, o sistema de corpo de bomba não previa a instalação de um dispositivo acessório, dessa forma não foi encontrado na tubulação nenhum orifício compatível com a bitola do sensor, $10 \mathrm{~mm}$, sendo necessário a utilização da saída do manômetro principal situado na tubulação direita do complexo. Ainda assim,necessitou-setrocar a conexão niple redutora de 1/2' para 9/16" por um tipo "T" (Figura 7A) onde contempla mais uma saída de $10 \mathrm{~mm}$ a qual foi rosqueado o sensor de temperatura.

Ainda discorrendo sobre os locais de instalação, o monitor, dispositivo que nos permite visualizar a temperatura da água, ficou acondicionado dentro do abrigo do mangotinho (Figura 7B) afim de evitar que sofra as consequências das ações de intempéries naturais. Contudo, o operador do corpo de bomba conseguiu visualizá-lo nitidamente.

Figura 7 - Posição da instalação do sensor. A) Sensor de Temperatura.

B) Monitor
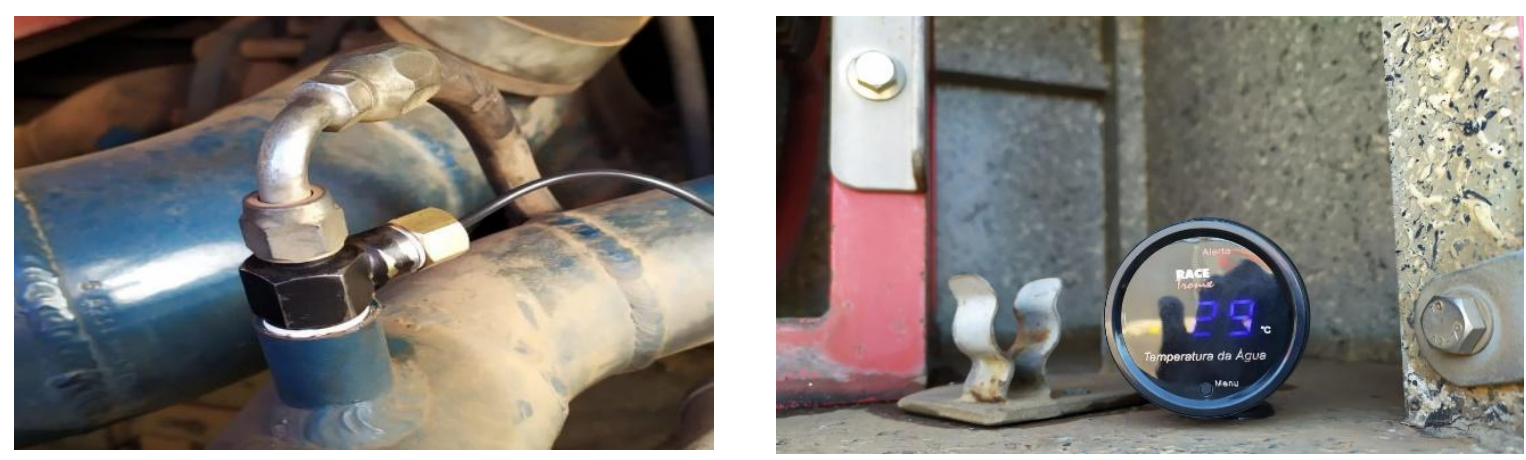

Fonte: Pesquisar

\subsection{Da Coleta de Dados}


Revista Científica do Corpo de Bombeiros Militar de Pernambuco

Artigo Publicado no Vol.06 N.15 - Edição Jan a Jun 2020 - ISSN 2359-4829

Versão on-line disponível em: http://www.revistaflammae.com

Os dados obtidos nos três experimentos, contemplando os tempos decorrente para aumento da temperatura, pressões e vazões, imprimida na bomba, bem como a rotação do motor e o volume do fluido no reservatório estão descritos na Tabela 1. Os resultados mostraram acréscimo progressivo da temperatura, onde foi possível observar um aumento de $5^{\circ} \mathrm{C}$ após 14 minutos e 35 segundos de funcionamento da bomba.

Um dos pontos principais a ser levado em consideração ao verificar a temperatura do sistema é o local que foi coletada essa informação. Portanto, o local de instalaçãodo sensor foi crucial para que os dados não fossem comprometidos. O ideal seria instalar o sensor de temperatura próximo ao rotor da bomba centrífuga, uma vez que, é o local mais aquecido e que mais sofre as consequências desse aumento térmico.

Tabela 1 Variação da temperatura e pressão em função do tempo

\begin{tabular}{|c|c|c|c|c|}
\hline $\begin{array}{l}\text { TESTE } 01 \\
\text { Horário }\end{array}$ & $\begin{array}{l}\text { Horário Inicial } \\
\text { Temperatura }\left({ }^{\circ} \mathrm{C}\right)\end{array}$ & $\begin{array}{c}10: 39: 00 \\
\text { Pressão (PSI) }\end{array}$ & $\begin{array}{c}\text { Volume Inicial } \\
\text { Vazão (LPM) }\end{array}$ & $\begin{array}{c}5000 \text { L } \\
\text { Giro (RPM) }\end{array}$ \\
\hline 00:00:00 & 25 & 25 & 500 & 700 \\
\hline 00:01:00 & 26 & 150 & 2850 & 1700 \\
\hline $00: 02: 38$ & 27 & 150 & 2850 & 1700 \\
\hline $00: 06: 25$ & 28 & 150 & 2850 & 1700 \\
\hline $00: 09: 45$ & 29 & 100 & 1990 & 1250 \\
\hline $00: 14: 35$ & 30 & 100 & 1990 & 1250 \\
\hline $00: 18: 45$ & 30 & 0 & 0 & 0 \\
\hline $\begin{array}{c}\text { TESTE } 02 \\
\text { Horário }\end{array}$ & $\begin{array}{c}\text { Horário Inicial } \\
\text { Temperatura }\left({ }^{\circ} \mathrm{C}\right)\end{array}$ & $\begin{array}{c}\text { 13:51:00 } \\
\text { Pressão (PSI) }\end{array}$ & $\begin{array}{l}\text { Volume Inicial } \\
\text { Vazão(LPM) }\end{array}$ & $\begin{array}{c}4600 \mathrm{~L} \\
\text { Giro(RPM) }\end{array}$ \\
\hline $00: 00: 00$ & 29 & 25 & 500 & 700 \\
\hline 00:01:00 & 30 & 150 & 2850 & 1700 \\
\hline 00:03:40 & 31 & 150 & 2850 & 1700 \\
\hline $00: 06: 45$ & 32 & 150 & 2850 & 1700 \\
\hline 00:10:00 & 33 & 150 & 2850 & 1700 \\
\hline $00: 13: 15$ & 34 & 150 & 2850 & 1700 \\
\hline $00: 16: 10$ & 35 & 150 & 2850 & 1700 \\
\hline 00:18:00 & 35 & 0 & 0 & 0 \\
\hline $\begin{array}{l}\text { TESTE } 03 \\
\text { Horário }\end{array}$ & $\begin{array}{c}\text { Horário Inicial } \\
\text { Temperatura }\left({ }^{\circ} \mathrm{C}\right)\end{array}$ & $\begin{array}{c}\text { 15:00:00 } \\
\text { Pressão (PSI) }\end{array}$ & $\begin{array}{l}\text { Volume Inicial } \\
\text { Vazão (LPM) }\end{array}$ & $\begin{array}{c}\text { 4200L } \\
\text { Giro (RPM) }\end{array}$ \\
\hline 00:00:00 & 34 & 25 & 500 & 700 \\
\hline $00: 01: 30$ & 35 & 150 & 2850 & 1700 \\
\hline $00: 03: 15$ & 36 & 150 & 2850 & 1700 \\
\hline
\end{tabular}




\begin{tabular}{ccccc}
\hline $00: 06: 00$ & 37 & 150 & 2850 & 1700 \\
$00: 09: 00$ & 38 & 150 & 2850 & 1700 \\
$00: 11: 35$ & 39 & 150 & 2850 & 1700 \\
$00: 14: 10$ & 150 & 2850 & 1700 \\
$00: 16: 50$ & 40 & 150 & 2850 & 1700 \\
$00: 17: 40$ & 41 & 0 & 0 & 0 \\
\hline
\end{tabular}

No entanto, como os testes foram feitos durante as instruções nos contêineres, atividades que coloca o corpo de bombas da viatura em situações extremas de funcionamento,já é utilizado um sistema de recirculação da água,conectando uma mangueira da saída de 2"1/2 ao reservatório. Os valores referentes às temperaturas foram coletados nessa tubulação de retorno., sendo importante enfatizar que, dessa forma, toda a água do reservatório também foi objeto de análise.

Ainda discorrendo sobre o momento da coleta de informações, esta aconteceu, como já citado acima, no instante em que a referida viatura estava empregada na instrução de flash over. Essa técnica, um tanto quanto nova na instituição, tem sido objeto de pesquisa, sobretudo no que diz respeitos às bombas centrifugas. Pois, apesar de ser eficiente no combate a incêndio, trabalha com uma vazão muito baixa e, portanto, tem-se um aumento significante de temperatura.

\subsection{Análise daVazão e Pressão}

A partir dos dados fornecidos pelo fabricante e utilizando a seguinte equação: valor total da vazão multiplicado por $80 \%$. Estes gráficos mostram o ponto de melhor eficiência (BEP)de vazão em bombas centrífugas de simples estágio (Gráfico 1A) e o ponto real de trabalho (PRT) dado pela vazão obtida na ponta da mangueira no referido experimento (Gráfico 1B). 
Revista Científica do Corpo de Bombeiros Militar de Pernambuco

Artigo Publicado no Vol.06 N.15 - Edição Jan a Jun 2020 - ISSN 2359-4829

Versão on-line disponível em: http://www.revistaflammae.com

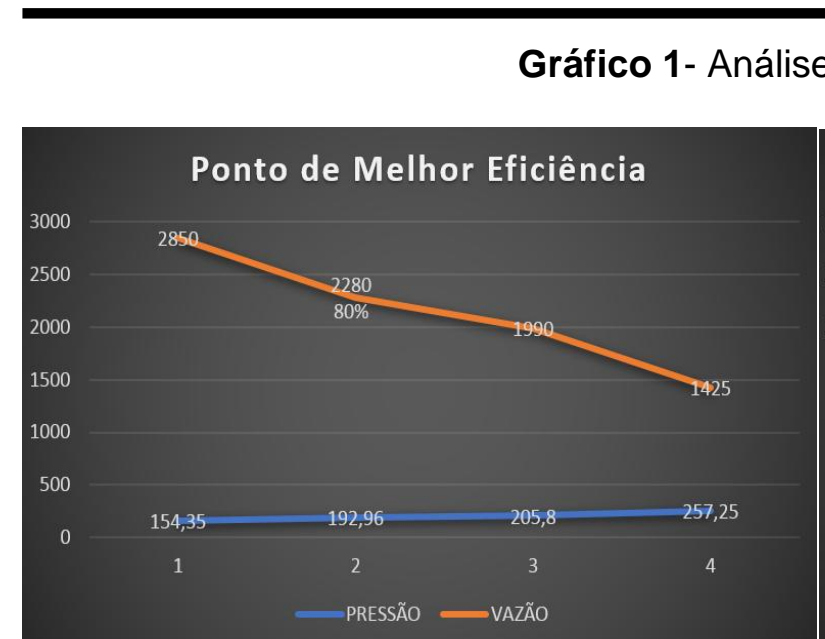

A) BEP $80 \%$

Fonte: Resultado da Pesquisa

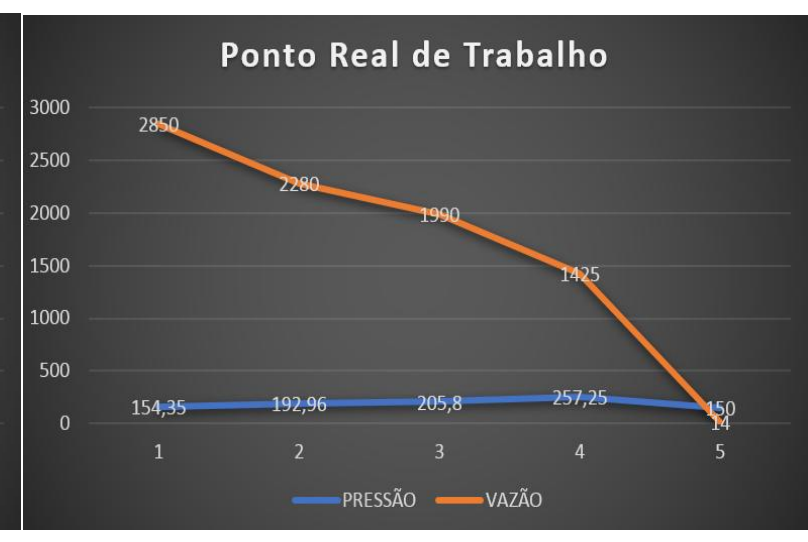

B)PRT $0,49 \%$

Ao analisar o gráfico $1 \mathrm{~A}$, visualiza-se que a bomba empregada no experimento tem vazão máxima 2850 litros por minuto. Contudo, os estudos de Gouvea (2008), mostraram que para a maioria das bombas centrifugas de simples ação, é fundamental que trabalhe com uma vazão de 70 a $85 \%$ da máxima, neste caso 2280litros por minuto. Já o gráfico 1B mostra que a vazão na ponta da mangueira a uma pressão de 150 PSI é aproximadamente 14 litros por minuto. Isso significa que o fluxo de água que passa pela bomba e é expelido para fora do sistema não é suficiente para resfriar o sistema.Para concluir essa análise, é importante dizer que como a viatura em questão não tem uma tubulação própria para recirculação da água, com finalidade de retardar o aquecimento, utilizou-se um sistema com mangueira de 2"1/2, este já citado no tópico metodologia. Porém, para atender a demanda de pressão na linha de combate, utilizando como retorno uma mangueira de 2"1/2 é necessário imprimir maior giro ao motor. Dessa forma, aumenta-se a quantidade de energia térmica produzida pela bomba e, consequentemente, a temperatura do líquido bombeado. 
Revista Científica do Corpo de Bombeiros Militar de Pernambuco

Artigo Publicado no Vol.06 N.15 - Edição Jan a Jun 2020 - ISSN 2359-4829

Versão on-line disponível em: http://www.revistaflammae.com

\subsection{Análise da Temperatura}

O comportamento da temperatura no corpo de bomba em relação a pressão e vazão imprimida no sistema também foram analisadosem três etapas (Gráficos 2), mostrando um aumento considerável de temperatura.

Gráfico 2 - Análise da temperatura

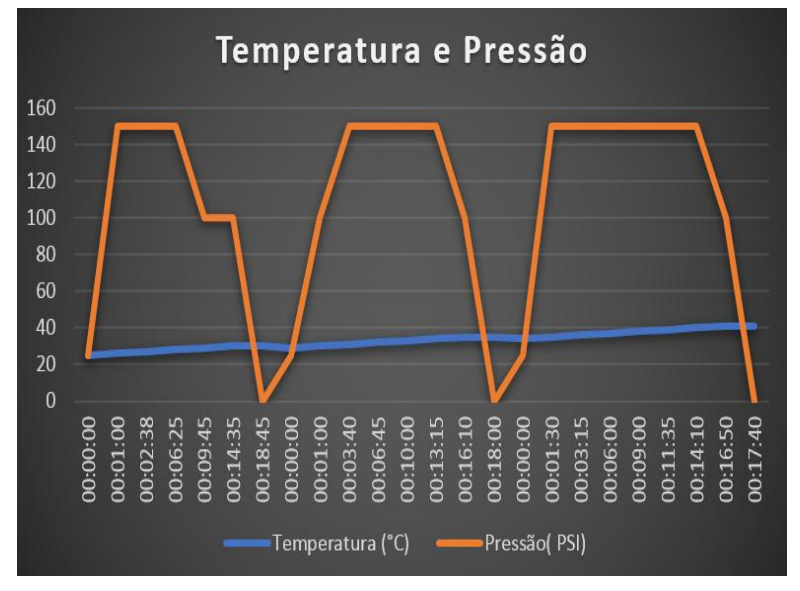

A)Temperatura versus Pressão

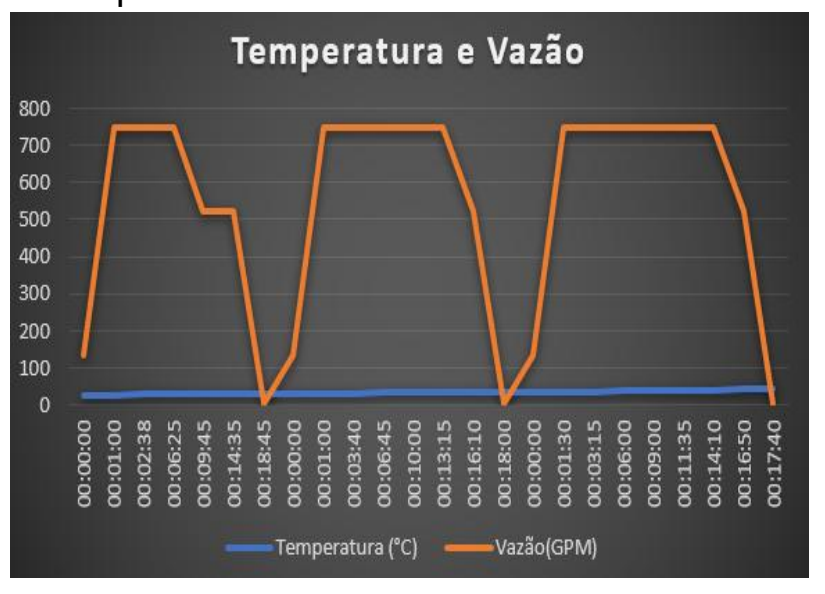

B)Temperatura versus Vazão

Fonte: Resultado de Pesquisa

A partir do gráfico $2 \mathrm{~A}$, foi possível observar que os dados começaram a serem contados desde o início do funcionamento da bomba, ou seja,25psi e nos próximos instantes é elevada à 150psi, permanecendo assim durante a primeira etapa. Nota-se que a temperatura foi crescente do início até o instante enquanto a pressão estava constante, do mesmo modo, tornou-se decrescente quando a pressão começou a ser reduzida.

Dessa maneira, fica evidente que a pressão tem uma relação direta com a temperatura. Ou seja,para aumentar a pressão no sistema cuja variáveis permaneceram constantesfoi necessário acrescentar giro ao motor, resultando em maior força sobre a área nas tubulações. Contudo, ao imprimir mais rotação, gerou-se mais atrito e consequentemente aquecimento. 
Por outro lado, enquanto o sistema de bombeamento foi aquecido, por consequência do atrito, o fluxo d'água que passou pela bomba não foi suficiente para que houvesse resfriamento, dado que a vazão com que 0 líquido foi expulso do sistema era muito baixa. No entanto, para que isso ocorresse com eficiência necessitava-se que a vazão expelida do sistema estivesse na faixa de $80 \%$ da capacidade máxima da referida bomba.

Entretanto, isso não acontece quando a técnica utilizada no combate é o flash over, uma vez que a vazão utilizada, exposta no gráfico $2 \mathrm{~B}$, foi muito baixa, cerca de $0,49 \%$ da máxima,não sendo suficiente para absorver o calor gerado pela bomba. Além disso, constatou-se que a vazão, nesse intervalo, esteve diretamente proporcional à pressão. Contudo, devido a água estar circulando internamente no sistema, passando pela bomba e a maior parte sendo devolvida ao reservatório, a mesma água cuja função era de resfriamento não foi capaz de cumprir seu papel, uma vez que todo o líquido contido no reservatório de 5 mil litros sofreu aquecimento.

A partir dos Gráficos 2A-B, também foi possível observar uma alta variação de temperatura, onde em dezoitos minutos de funcionamentoa temperatura aumentou $8^{\circ} \mathrm{C}$ e dentro de 45 minutos o fluido chegou a $41^{\circ} \mathrm{C}$. Contudo, até a temperatura de $41^{\circ} \mathrm{C}$ não houve a identificação de ruídos, vibrações ou quaisquer outros alertas de cavitação, permitindo que o operador do sistema possa deixá-lo trabalhar até essa temperatura sem que seja causado danos ao equipamento.

\subsubsection{Estimativa da Temperatura em Função do Tempo e Segurança do Combatente.}

Por meio de média aritmética dos tempos computados, foi estimado o comportamento da temperatura (Gráfico 3). Nota-se que num espaço de aproximadamente três horas o líquido sai da temperatura ambiente $\left(25^{\circ} \mathrm{C}\right)$ chegando ao ponto de ebulição a pressão de $1 \mathrm{~atm}$. Com isso, nota-se que o 
tempo de elevação da temperatura foi relativamente baixo, sendo de fundamental importância que o operador do corpo de bomba atente-se ao monitor, pois dessa forma evitará danos ao sistema mecânico como, também, protegerá o combatente que tem contato direto com a água aquecida.

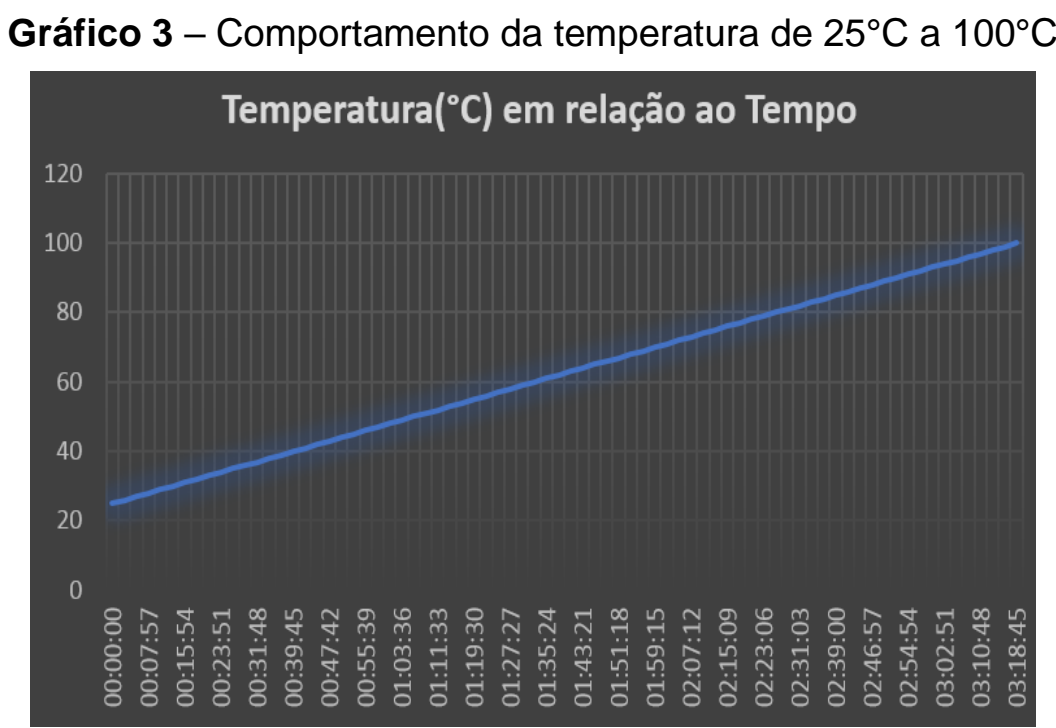

Fonte: Resultado de Pesquisa

\subsection{Influência da Temperatura àSerpentina}

Como discutido no referencial bibliográfico, o sistema de trocador de calor utilizados nestas caixas de transferências para manter o óleo a temperatura de trabalho é do tipo serpentina e precisa que a temperatura da água seja inferior à do óleo. Nas circunstâncias atuais, a serpentina tem sua eficiência parcialmente comprometida, pois assim como o fluido lubrificante e resfriador das engrenagens da caixa aquecem, o líquido que tem a função de absorver esse calor também esquenta, este devido à baixa vazão de trabalho. 


\section{CONSIDERAÇÕES FINAIS}

O monitoramento da temperatura no sistema de bombas da viatura de combate a incêndio (ABT-26), mostrou-se eficiente, pois:

- O sistema de monitoramento demostrou-se eficiente, permitindo ao operador ter ciência da temperatura em tempo real.

- Fica comprovado que existe, quando utilizada a técnica Flash over, um aumento considerável da temperatura decorrente do baixo fluxo de água expelido do sistema.

- Constatou-se, que até a temperatura de $41^{\circ} \mathrm{C}$ pode se trabalhar normalmente sem que haja danos ao corpo de bombas.

- Atividades na qual requer mais de 45 minutos, recomenda-se que seja aumentado o volume de água circulante ou que se faça intervalos, completando o nível do fluido e, consequentemente, reduzindo a temperatura.

- Com o aumento da temperatura da água, o sistema de arrefecimento da caixa de transferência é parcialmente comprometido. No entanto, Recomenda-se para este um radiador de óleo e um sensor de temperatura com monitor no painel de operações.

- Recomenda-se tubulações própria para um sistema de retorno.

\section{REFERÊNCIAS BIBLIOGRÁFICAS}

CARNEIRO, André. Sensores de Temperatura. Disponível em $<$ http://www.uesc.br/centros/ctr/modulos/didatico/palestras/seminarios/sct_2009 /sem_dia3_andre.pdf >. acesso em: 03 abr. 2019.

LARANJEIRA, Dan Godoy. Análise dos testes de performace e cavitação em uma bomba centrífuga. Universidade Federal do Rio de Janeiro, 2017. 
FEGHALI, Artur Kasznar. Análise de cavitação em bombas de alimentação de caldeiras de uma fábrica de bebidas e projeto de solução. Universidade Federal do Rio de Janeiro, 2016.

FRASER, Warrern H. Recirculação em bombas centrífugas. Revista DAE, 1981.

GOUVEA, Marcos Martins Rezende. Estudo de confiabilidade em bombas centrífugas. Universidade de São Francisco. 2018.

GOMES, Gustavo Alvez Pinto Mosqueira. Seleção de bomba centrífuga vertical para operação em um sistema de transferência de enxofre líquido. Universidade Federal do Rio de Janeiro. 2013.

MELO, Weber Bizarrias de. Efeitos da pressão do sistema de arrefecimento e da concentração de etileno glicol sobre as características de cavitação de uma bomba d'água automotiva. Escola Politécnica da Universidade de São Paulo. 2008.

MITREN. Manual de Operação: Bomba Centrífuga de Incêndio. Santa Cruz do Sul, 2016.Disponível em: assistênciatecnica@mitren.com.br>. Acesso em: 24 jul.2019.

OLIVEIRA, Felipe Holmann. Análise de vibração de eixos em bombas hidráulicas centrífugas. Universidade Tecnológica Federal do Parana. 2017.

RECETRONIX. Manual de Instalação e Programação. Bauru,2019. Disponível em:<https://www.racetronix.com.br/INDEX Agua.htm>. Acesso em: 30 jul. 2019.

SILVA, Hoberdan Batista. Montagem de um controlador de temperatura usando termopar.Universidade Federal de Uberlândia. 2006.

SOUZA. Pedro Henrique A. I. de. Apresentação dos cálculos para seleção de bomba para Sistema de reaproveitamento de água de poços artesianos. Universidade Federal do Rio de Janeiro. 2014.

SOUZA, Monique Silveira. Análise térmica de um trocador de calor do tipocasco e tubos para resfriamento do resíduo de uma unidade de destilação atmosférica. Universidade Federal do Rio de Janeiro. 2013. 